Case Reports

\title{
Clinical Case Report of a Novel Immunotherapeutic Approach to Autism Spectrum Disorders based on an emulsion of Chondroitin Sulfate, Vitamin $D_{3}$ and Oleic Acid
}

\author{
${ }^{1}$ Nicola Antonucci, ${ }^{2}$ Stefania Pacini and ${ }^{2}$ Marco Ruggiero \\ ${ }^{1}$ Biomedical Centre for Autism Research and Treatment, Bari, Italy \\ ${ }^{2}$ Silver Spring Sagl, Arzo-Mendrisio, Switzerland
}

Article history

Received: 24-07-2017

Revised: 1-08-2017

Accepted: 7-08-2017

Corresponding Author:

Stefania Pacini

Silver Spring Sagl, Arzo-

Mendrisio, Switzerland

Email: pacini68@yahoo.com

\begin{abstract}
In this clinical case report we describe the effects associated with the administration of a supplement constituted by chondroitin sulfate, vitamin $\mathrm{D}_{3}$ and oleic acid $\left(\right.$ Rerum $\left.^{\circledR}\right)$ on the symptoms of Autism Spectrum Disorders (ASD). 44 patients (11 females and 33 males) were administered this supplement for a period of two months and variations of symptomatology were evaluated by the Clinical Global Impression of Improvement (CGI-I). 32 out of 44 subjects $(73 \%)$, showed improvements in the two months of observation. In the group of female subjects, 9 out of $11(81 \%)$ showed improvements and, among these, 6 out of $9(66 \%)$ showed moderate to very considerable improvements. In the group of male subjects, 24 out of 33 (73\%) showed improvements and, among these, 22 out of 24 (91\%) showed moderate to very considerable improvements. We attribute these remarkable results to the effects of the supplement on the endocannabinoid system as it relates to neuro-inflammation and neuro-immune alterations.
\end{abstract}

Keywords: Autism, Endocannabinoid, GcMAF, Chondroitin Sulfate, Vitamin $\mathrm{D}_{3}$, Oleic Acid

\section{Introduction}

The term Autism Spectrum Disorders (ASD) encompasses a wide range of heterogeneous alterations of neural development characterized by three basic features: Impaired social interaction, language and communication abnormalities and stereotypical behavior. Although the etiology of ASD is debated and still largely unclear (Yenkoyan et al., 2017; Geier et al., 2017), the pathogenesis seems to be associated with alterations of the immune system and neuro-inflammation. Thus, we recently hypothesized that infections or inflammation at the level of the deep cervical nodes lead to impaired lymphatic drainage from the brain lymphatic system with consequent stagnation of lymph in the brain, disruption of neuronal connections, accumulation of metabolites and toxicants, neuroinflammation and alterations of the brain intrinsic microbiota (Bradstreet et al., 2015; Ruggiero, 2016a). Because of the heterogeneity of the symptoms and the lack of certainty surrounding the cause(s), it is no surprise that the very existence of a standard of care for ASD is highly disputed and even reviews of systematic reviews fail to provide useful information that may help health providers working in the frontlines of this growing epidemics (Lyra et al., 2017).

At the Biomedical Centre for Autism Research and Treatment, several therapeutic strategies targeting the immune system and neuro-inflammation have been implemented over the course of the years (Antonucci et al., 2015). In the recent past, we focused our attention on the role of modulators of the immune system as potential candidates for ASD treatment based on the seminal work by Bradstreet et al. (2012) on the Gc protein-derived Macrophage Activating Factor (GcMAF). Since it was recently hypothesized that the results observed by Bradstreet et al. (2012) may be due to a glycosaminoglycan, chondroitin sulfate (Ruggiero et al., 2016; Ruggiero, 2016b), we decided to treat ASD subjects with a chondroitin sulfate-containing supplement that has shown remarkable results in the field of immunotherapy (Schwalb et al., 2016; Ruggiero, 2017). Here we report the first series of results observed in 44 ASD subjects treated for two months with this supplement constituted by chondroitin sulfate, vitamin $\mathrm{D}_{3}$ and oleic acid $\left(\right.$ Rerum $\left.^{\circledR}\right)$. 


\section{Patients and Methods}

The immunotherapeutic approach described in this study is similar, in line of principle, to that described in Schwalb et al. (2016), that is an approach currently implemented in a number of Clinics. The original clinical records as well as the informed consents given by patients or their parents or legal guardians, are conserved at the Biomedical Centre for Autism Research and Treatment. In the clinical case report described here, 44 patients (11 females and 33 males) were administered the emulsion of chondroitin sulfate, vitamin $\mathrm{D}_{3}$ and oleic acid mentioned in the Introduction and thoroughly described in a recent paper (Ruggiero, 2017). Such an emulsion, marketed as Rerum $^{\circledR}$, is manufactured in Germany by the company "dr. reinwald healthcare" and it is classified and registered in Europe and Switzerland as a food supplement since it is composed by well-known supplements that have been in use for decades. Although supplements are usually intended to be administered through the oral route, according to national rules and regulations and under the direct responsibility of the Therapist, they can also be administered through the parenteral route as described in Schwalb et al. (2016). The period of observation to which this study refers to spanned two years (2016 and 2017) and each patient was observed for two months. All patients met the diagnostic criteria for ASD and were diagnosed by either a child neurologist or developmental psychologist, in addition to receiving the evaluation of the clinician (NA). No significant changes were made to the participants' treatments apart from the introduction of Rerum $^{\circledR}$ during the timeframe reported in this study. The initial dose for each patient was $0.01 \mathrm{~mL}$ per week. In some subjects, the dose was progressively adjusted to the body weight up to the maximum tolerated dose or up to the minimum efficient dose.

In addition to meeting diagnostic criteria for ASD, the clinician supervising this study (NA) used an in-house severity scoring system that is based on the Clinical Global Impression of Improvement (CGI-I). According to this system, the score was as designated follows:

5: Very considerable improvement

4: Considerable improvement

3: Moderate improvement

2: Slight improvement

1: Very slight improvement

0 : State unchanged

-1 : Very slight deterioration

-2 : Slight deterioration

-3: Moderate deterioration

-4: Considerable deterioration

-5 : Very considerable deterioration

-6: Maximum deterioration

Age, gender, body weight and CGI-I score of participants are summarized in Table 1.

\section{Results}

Table 1 and Fig. 1 (upper panel) show that 32 out of 44 subjects $(73 \%)$ showed improvements in the two months of observation. Of these, 28 out of $32(87 \%)$ showed moderate to very considerable improvements. In the group of those who showed improvements, 16 subjects (50\%) showed significant or very significant improvement. In the group of female subjects (Fig. 1, middle panel), 9 out of $11(81 \%)$ showed improvements and, among those who showed improvements, 6 out of 9 (66\%) showed moderate to very considerable improvements. In the group of male subjects (Fig. 1, lower panel), 24 out of $33(73 \%)$ showed improvements and, among those who showed improvements, 22 out of 24 (91\%) showed moderate to very considerable improvements.

Table 1. Age, gender, body weight and CGI-I score

\begin{tabular}{|c|c|c|c|c|}
\hline Subject & Age & Gender & Body weight $(\mathrm{Kg})$ & CGI-I \\
\hline 1 & 5.5 & $\mathrm{M}$ & 23.0 & 5 \\
\hline 2 & 5.0 & M & 23.0 & 5 \\
\hline 3 & 9.0 & M & 53.0 & 5 \\
\hline 4 & 5.0 & $\mathrm{~F}$ & 19.0 & 4 \\
\hline 5 & 5.3 & $\mathrm{~F}$ & 15.0 & 4 \\
\hline 6 & 6.0 & M & 24.0 & 4 \\
\hline 7 & 5.5 & M & 20.0 & 4 \\
\hline 8 & 4.5 & M & 15.5 & 4 \\
\hline 9 & 3.0 & M & 19.0 & 4 \\
\hline 10 & 3.8 & M & 15.0 & 4 \\
\hline 11 & 6.5 & M & 20.0 & 4 \\
\hline 12 & 6.5 & M & 20.0 & 4 \\
\hline 13 & 5.0 & M & 21.0 & 4 \\
\hline 14 & 3.0 & M & 14.5 & 4 \\
\hline 15 & 4.0 & M & 17.5 & 4 \\
\hline 16 & 4.0 & M & 20.0 & 4 \\
\hline 17 & 14.0 & $\mathrm{~F}$ & 40.0 & 3 \\
\hline 18 & 4.5 & $\mathrm{~F}$ & 24.0 & 3 \\
\hline 19 & 7.5 & F & 24.0 & 3 \\
\hline 20 & 6.5 & $\mathrm{~F}$ & 30.0 & 3 \\
\hline 21 & 4.5 & $\mathrm{M}$ & 20.0 & 3 \\
\hline 22 & 6.0 & M & 20.0 & 3 \\
\hline 23 & 6.0 & $\mathrm{M}$ & 16.0 & 3 \\
\hline 24 & 2.5 & $\mathrm{M}$ & 14.0 & 3 \\
\hline 25 & 4.5 & $\mathrm{M}$ & 17.0 & 3 \\
\hline 26 & 5.0 & $\mathrm{M}$ & 24.0 & 3 \\
\hline 27 & 4.0 & $\mathrm{M}$ & 16.0 & 3 \\
\hline 28 & 6.5 & M & 20.0 & 3 \\
\hline 29 & 3.0 & $\mathrm{~F}$ & 15.0 & 2 \\
\hline 30 & 4.0 & $\mathrm{~F}$ & 14.5 & 2 \\
\hline 31 & 3.0 & $\mathrm{M}$ & 13.0 & 2 \\
\hline 32 & 4.5 & $\mathrm{M}$ & 21.0 & 2 \\
\hline 33 & 4.0 & $\mathrm{~F}$ & 16.0 & 1 \\
\hline 34 & 2.5 & $\mathrm{M}$ & 14.0 & 0 \\
\hline 35 & 3.5 & M & 11.5 & 0 \\
\hline 36 & 3.0 & M & 15.0 & 0 \\
\hline 37 & 9.0 & M & 36.0 & 0 \\
\hline 38 & 3.0 & $\mathrm{M}$ & 14.0 & 0 \\
\hline 39 & 3.5 & M & 18.0 & 0 \\
\hline 40 & 3.0 & M & 13.5 & 0 \\
\hline 41 & 3.0 & M & 16.0 & 0 \\
\hline 42 & 5.5 & $\mathrm{~F}$ & 19.0 & -1 \\
\hline 43 & 11.0 & M & 51.0 & -1 \\
\hline 44 & 3.0 & $\mathrm{~F}$ & 18.0 & -4 \\
\hline
\end{tabular}


Upper panel

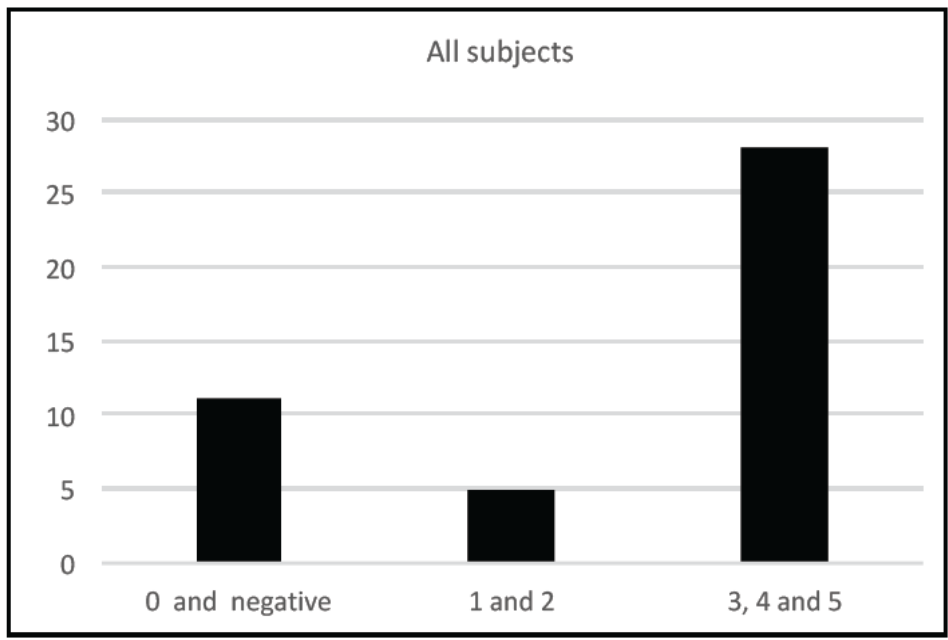

Middle panel

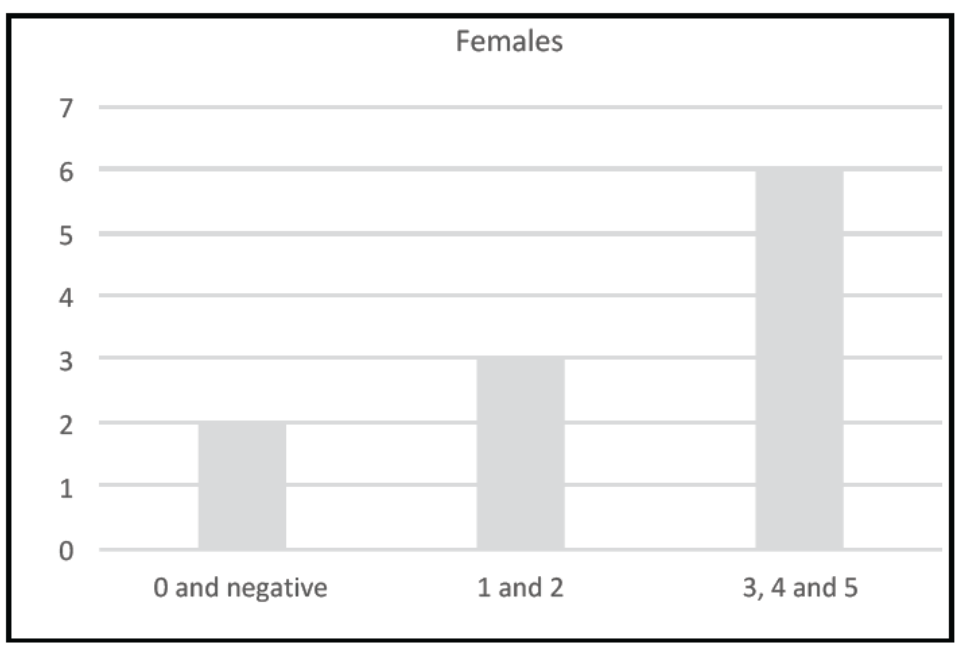

\section{Lower panel}

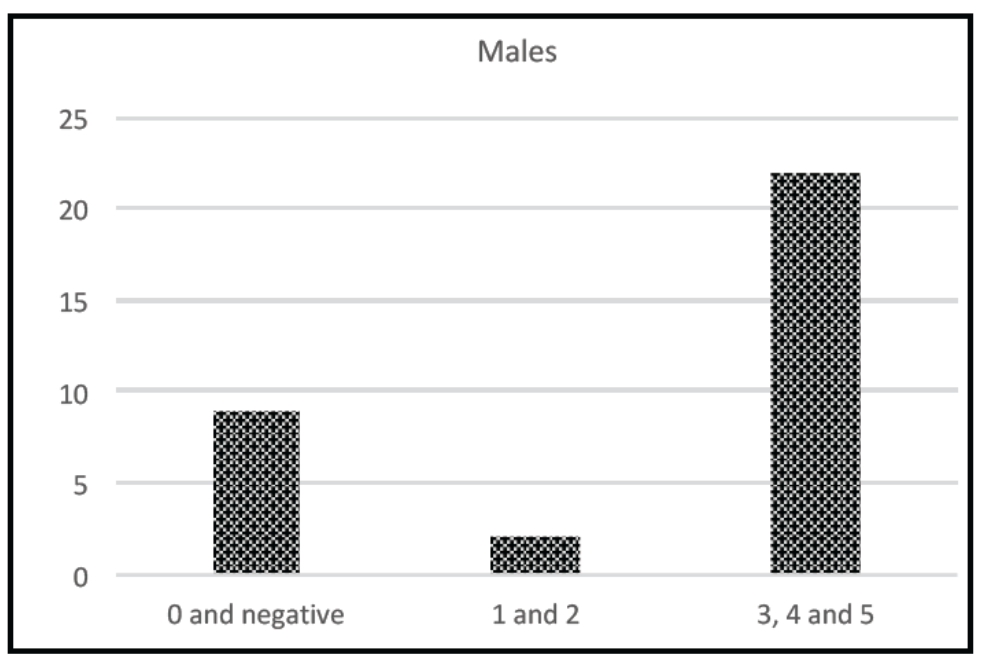

Fig. 1. Clinical Global Impression of Improvement (CGI-I) responses. Y-axis represents the number of subjects in each group. 0 and negative, indicates subjects who showed no improvements or deterioration. 1 and 2 indicates subjects who showed very slight or slight improvements. 3-5 indicates subjects who showed moderate, considerable or very considerable improvements. Upper panel refers to the entire group of subjects; middle panel refers to female subjects; lower panel refers to male subjects 
The 3 subjects who showed very significant improvements (CGI-I score 5) belonged to the group of male subjects. In the group of 12 subjects who showed significant improvements (CGI-I score 5), 10 $(83 \%)$ were males. In the group of those subjects who did not show any improvement 9 out of 11 were males. We could not find association between the degree of improvement and age or body weight.

\section{Discussion}

Since this clinical case report is an open-label, noncontrolled, retrospective analysis, caution must be employed when ascribing cause and effect to any treatment outcome. However, the response to Rerum ${ }^{\circledR}$ was robust with regard to symptomatic improvements as shown by the CGI-I score and we attribute these remarkable results to the effects of the supplement on the Endocannabinoid (EC) system as it relates to neuroinflammation and neuro-immune alterations. Thus, we recently described the role of the EC system in the pathophysiology of ASD and we highlighted the involvement of the EC system in autism-associated immunological disruptions (Brigida et al., 2017). Preliminary results obtained in our laboratory seem to indicate that $\operatorname{Rerum}^{\circledR}$ acts on the EC system in vitro (manuscript in preparation). This is not surprising considering that the components of Rerum ${ }^{\circledR}$ are known to interact with the metabolisms of 2-arachidonoyl glycerol (2-AG), the physiological ligand for both cannabinoid receptors CB1 and CB2 (Sugiura et al., 1999; Gonsiorek et al., 2000). Thus, 2-AG derives from the hydrolysis of 1,2-Diacylglycerol (DAG) (Reisenberg et al., 2012), a second messenger that is formed either as consequence of phospholipase $\mathrm{C}$ hydrolysis of inositol lipids or phosphatidylcholine (Michell, 1983; Lacal, 1990), or as consequence of de novo synthesis through the glycolytic pathway (Chiarugi et al., 1989). More than thirty years ago, we demonstrated that glycosaminoglycans similar in structure to chondroitin sulfate interact with the metabolism of inositol lipids (Ruggiero et al., 1984) or phosphatidylcholine (Vannucchi et al., 1985), thus leading to DAG and 2-AG endogenous production. Also the other component of Rerum ${ }^{\circledR}$, vitamin $D$, is known to stimulate the formation of DAG through stimulation of inositol lipid metabolism (Doroudi et al., 2012), thus converging on the same EC pathway thanks to the production of 2-AG from DAG.

It is acknowledged that the EC system is a promising therapeutic target in the field of ASD research (Yeh and Levine, 2017) and our observations seem to indicate that Rerum $^{\circledR}$, by stimulating the endogenous production of 2AG, may play a role in this field.

\section{Conclusion}

A recent systematic review of the scientific literature states that "the use of dietary supplements in children with autism seems to be a safe practice with encouraging data about their clinical efficacy" (Gogou and Kolios, 2017). Our observations appear to be consistent with this statement in that they suggest that the use of Rerum $^{\circledR}$, a supplement composed of chondroitin sulfate, vitamin $\mathrm{D}_{3}$ and oleic acid, may be associated with significant improvements of the symptoms of the disease.

\section{Acknowledgement}

The Authors wish to thank Dr. Heinz Reinwald and Kerstin Reinwald for their contribution to the manufacture of Rerum ${ }^{\circledR}$ and for the organization of the Fourth International Congress on Integrative Medicine that was held in Fulda, Germany, on April 1 and 2, 2017.

\section{Author's Contributions}

Nicola Antonucci: Performed all the diagnostic and therapeutic procedures described in this study.

Marco Ruggiero and Stefania Pacini: Wrote the first draft of this paper, provided critical input and assisted in revising and improving the paper.

\section{Conflict of Interest}

Nicola Antonucci is the founder of the Biomedical Centre for Autism Research and Treatment, a private clinic.

Marco Ruggiero is the inventor of Rerum ${ }^{\circledR}$ and consults for the company "dr. reinwald healthcare". He had no prior knowledge of the therapies being used nor of the names of any patient whose data were being analyzed and described in this study. Neither he, nor any employee of company "dr. reinwald healthcare", had any prior knowledge of the clinical records or the patients' names used in this study. Marco Ruggiero is member of the Editorial Board of The American Journal of Immunology and is waived from the Article Processing fee for this contribution; he receives no remuneration for his editorial work.

Stefania Pacini declares no conflict of interest.

\section{Ethics}

This article is original and contains material that has not been published in any other scientific journal. Preliminary reports of some of the results described in this study have been presented at the Fourth International Conference on Integrative Medicine (Fulda, Germany, April 2017) and at the AutismOne 2017 Conference (Colorado Springs, USA, May 2017).The corresponding author confirms that the other authors have read and approved the manuscript. 


\section{References}

Antonucci, N., A. Cirillo and D. Siniscalco, 2015. Beneficial effects of palmitoylethanolamide on expressive language, cognition and behaviors in autism: A report of two cases. Case Rep. Psychiatry, 2015: 325061-325061. DOI: 10.1155/2015/325061

Bradstreet, J.J., E. Vogelaar and L. Tyer, 2012. Initial observations of elevated Alpha-NAcetylgalactosaminidase activity associated with autism and observed reductions from GC proteinmacrophage activating factor injections. Autism Insights, 4: 31-38. DOI: 10.4137/AUI.S10485

Bradstreet, J.J., M. Ruggiero and S. Pacini, 2015. Commentary: Structural and functional features of central nervous system lymphatic vessels. Front. Neurosci., 9: 485-485.

DOI: $10.3389 /$ fnins. 2015.00485

Brigida, A.L., S. Schultz, M. Cascone, N. Antonucci and D. Siniscalco, 2017. Endocannabinod signal dysregulation in autism spectrum disorders: A correlation link between inflammatory state and neuro-immune alterations. Int. J. Mol. Sci., 18: E1425-E1425. DOI: 10.3390/ijms18071425

Chiarugi, V., P. Bruni, F. Pasquali, L. Magnelli and G. Basi et al., 1989. Synthesis of diacylglycerol de novo is responsible for permanent activation and downregulation of protein kinase $\mathrm{C}$ in transformed cells. Biochem. Biophys. Res. Commun., 164: 816-823. DOI: $10.1016 / 0006-291 X(89) 91532-5$

Doroudi, M., Z. Schwartz and B.D. Boyan, 2012. Phospholipase $\mathrm{A}_{2}$ activating protein is required for 1 $\alpha, 25$-dihydroxyvitamin $\mathrm{D}_{3}$ dependent rapid activation of protein kinase $\mathrm{C}$ via Pdia3. J. Steroid Biochem., 132: 48-56. DOI: 10.1016/j.jsbmb.2012.03.001

Geier, D.A., J.K. Kern, K.G. Homme and M.R. Geier, 2017. Abnormal brain connectivity spectrum disorders following thimerosal administration: A prospective longitudinal case-control assessment of medical records in the vaccine safety datalink. Dose Response: Int. J. DOI: 10.1177/1559325817690849.

Gogou, M. and G. Kolios, 2017 The effect of dietary supplements on clinical aspects of autism spectrum disorder: A systematic review of the literature. Brain Dev., 39: 656-664. DOI: $10.1016 /$ j.braindev.2017.03.029

Gonsiorek, W., C. Lunn, X. Fan, S. Narula and D. Lundell et al., 2000. Endocannabinoid 2-arachidonyl glycerol is a full agonist through human type 2 cannabinoid receptor: Antagonism by anandamide. Mol. Pharmacol., 57: 1045-1050. PMID: 10779390

Lacal, J.C., 1990. Diacylglycerol production in Xenopus laevis oocytes after microinjection of p21ras proteins is a consequence of activation of phosphatidylcholine metabolism. Mol. Cell. Biol., 10: 333-340. DOI: 10.1128/MCB.10.1.333
Lyra, L., L.E. Rizzo, C.S. Sunahara, D.V. Pachito and C.O.C. Latorraca et al., 2017. What do Cochrane systematic reviews say about interventions for autism spectrum disorders? Sao Paulo Med. J., 135: 192-201. DOI: 10.1590/1516-3180.2017.0058200317

Michell, R.H., 1983. V. Polyphosphoinositide breakdown as the initiating reaction in receptorstimulated inositol phospholipid metabolism. Life Sci., 32: 2083-2085. DOI: 10.1016/0024-3205(83)90095-4

Reisenberg, M., P.K. Singh, G. Williams and P. Doherty, 2012. The diacylglycerol lipases: Structure, regulation and roles in and beyond endocannabinoid signalling. Phil. Trans. R. Soc. B, 367: 3264-3275. DOI: $10.1098 /$ rstb.2011.0387

Ruggiero, M, 2016a. Fecal microbiota transplantation and the brain microbiota in neurological diseases. Clin. Endosc., 49: 579-579.

DOI: $10.5946 /$ ce. 2016.098

Ruggiero, M., 2016b. Gc protein-derived Macrophage Activating Factor (GcMAF) and autism: Do clinical results require a novel interpretation?? Am. J. Immunol., 12: 85-90. DOI: 10.3844/ajisp.2016.77.82

Ruggiero, M., H. Reinwald and S. Pacini, 2016. Is chondroitin sulfate responsible for the biological effects attributed to the GC protein-derived Macrophage Activating Factor (GcMAF)? Med. Hypotheses, 94: 126-31.

DOI: 10.1016/j.mehy.2016.07.012

Ruggiero, M., S. Fedi, P. Bianchini, S. Vannucchi and V. Chiarugi, 1984. Molecular events involved in the proaggregating effect of heparin on human platelets. Biochim. Biophys. Acta., 802: 372-377. DOI: $10.1016 / 0304-4165(84) 90185-5$

Ruggiero, M., 2017. Is rerum the new Coley's vaccine? Am. J. Immunol., 2: 91-98.

DOI: 10.3844 ajisp.2017.91.98

Schwalb, M., M. Taubmann, S. Hines, H. Reinwald and M. Ruggiero, 2016. Clinical observation of a novel, complementary, immunotherapeutic approach based on ketogenic diet, chondroitin sulfate, vitamin $\mathrm{D}_{3}$, oleic Acid and a fermented milk and colostrum product.Am. J. Immunol., 12: 91-98. DOI: 10.3844 /ajisp.2016.91.98

Sugiura, T., T. Kodaka, S. Nakane, T. Miyashita and S. Kondo et al., 1999. Evidence that the cannabinoid $\mathrm{CB} 1$ receptor is a 2-arachidonoylglycerol receptor: Structure-activity relationship of 2arachidonoylglycerol, ether-linked analogues and related compounds. J. Biol. Chem., 274: 2794-2801. DOI: $10.1074 /$ jbc.274.5.2794 
Vannucchi, S., M. Ruggiero and V. Chiarugi, 1985. Complexing of heparin with phosphatidylcholine. A possible supramolecular assembly of plasma heparin. Biochem. J., 227: 57-65.

DOI: $10.1042 / b j 2270057$

Yeh, M.L. and E.S. Levine, 2017. Perspectives on the Role of Endocannabinoids in Autism Spectrum Disorders. OBM Neurobiol., 1: 005-005.

DOI: 10.21926/obm.neurobiol.1702005
Yenkoyan, K., A. Grigoryan, K. Fereshetyan and D. Yepremyan, 2017. Advances in understanding the pathophysiology of autism spectrum disorders. Behav. Brain. Res., 331: 92-101.

DOI: $10.1016 /$ j.bbr.2017.04.038 